\section{Fettfeuchte Verbände bei atopischem Ekzem} \section{Kortikoide steigern Effektivität}

\author{
Juckreiz und Entzündung sind die Hauptsymptome beim atopi- \\ schen Ekzem. Kühlung, z.B. durch fettfeuchte Verbände, lindert diese \\ Symptome. Kindern mit leichten Hautveränderungen kann so \\ bereits ohne weitere Medikation geholfen werden. Bei den mittel- \\ schweren und schweren Formen empfiehlt sich der Zusatz eines \\ Antiseptikums, oder, noch effektiver, eines modernen Kortikoids.
}

Fom euchte Umschläge, so D. Abeck (München), werden allgemein bei entzündeter Haut als angenehm empfunden: Die bei der Verdunstung entzogene Wärmeenergie führt zu einer $\mathrm{Ab}$ kühlung und lindert dadurch die Symptome. Um ein Austrocknen der Haut zu verhindern, wird diese vorher mit fetthaltigen Basistherapeutika versorgt.

Dieses Prinzip der fettfeuchten Verbände (,wet-wrap-dressing") hat sich nach Abecks Worten als ein wichtiger Therapiepfeiler im Management des atopischen Ekzems bewährt - vor allem in der Behandlung der überwiegend aufgekratzten, nässenden und häufig verkrusteten Hautveränderungen an Rumpf und Extremitäten.

\section{Verband wird gut toleriert}

Das Prozedere ist einfach und wird von den Kindern gut toleriert: Nach dem Einfetten der Haut werden die betroffenen Areale mit angefeuchteten, meist industriell gefertigten Bandagen umwickelt - eine Technik, die mit dem „feuchten Schlafanzug" als Maximalvariante auf den ganzen Körper ausgedehnt werden kann. Gleichzeitig stellt der Verband einen wirksamen Schutz vor eventuellen Kratzaktivitäten des Kindes dar.

Wird der Verband tagsüber getragen, dann meist über vier Stunden, kann das Kind seinen normalen Aktivitäten nachgehen. Viele Kinder tragen die Bandagen aber auch problemlos die ganze Nacht über.

Bei schweren Formen des atopischen Ekzems können dem feuchten Verband antiseptische Zubereitungen, z.B. eine $0,5 \%$ ige wässrige Chlorhexi-
din-Lösung, zugesetzt werden. Nach dreitägiger Behandlung verbessert sich dadurch der mikrobiologische Hautbefund der kleinen NeurodermitisPatienten deutlich. Denn ihre Haut ist - im Gegensatz zu der von Gesunden häufig mit Staphylococcus aureus besiedelt, was heute als erkrankungsrelevanter Provokationsfaktor angesehen wird.

\section{Reduzierte Bakterienbesiedlung}

Einen noch besseren therapeutischen Effekt erzielt man durch den Zusatz von topischen Glukokortikoiden. Dies ist angesichts der hohen Sicherheit moderner Substanzvertreter mit geringem atrophogenem Potenzial und gleichzeitig hoher antiinflammatorischer Wirksamkeit auch für Kinder absolut zu vertreten, so Abeck. Interessanter $\mathrm{Ne}-$ benbefund einer solchen Behandlung: In Abhängigkeit von der Stärke der entzündungshemmenden Wirkung des Kortikoids nimmt auch die Bakterienkonzentration auf der Haut ab.

\section{Mometason im Test}

An der Dermatologischen Klinik und Poliklinik der Technischen Universität München wird unter Federführung Abecks derzeit im Halbseitenvergleich gegen ein Basistherapeutikum Wirksamkeit und Verträglichkeit des modernen Glukokortikoids Mometasonfuroat $\left(\right.$ Ecural $\left.^{\circledR}\right)$ im Zusammenspiel mit der fettfeuchten Behandlung untersucht.

Neben der klinischen Dokumentation zu Beginn, nach drei Tagen und am Abschluss nach fünf Tagen werden zusätzlich bakteriologische Untersuchungen durchgeführt.

Erste Zwischenauswertungen, so Abeck, ergaben insbesondere beim schweren atopischen Ekzem deutliche Seitenunterschiede, die - obwohl die Verblindung der Studie noch nicht aufgehoben ist - auf eine Überlegenheit der Steroidbehandlung hinweisen. $\quad b k$
Abeck D
Pressesymposium der Essex Pharma, Petersberg bei Königswinter,
August 2000.

\section{Atopisches Ekzem multifaktoriell behandeln}

Der komplexen Pathogenese des atopischen Ekzems entsprechend muss auch deren Therapie an verschiedenen Punkten ansetzen, so T. Ruzicka (Düsseldorf). Ein Eckpfeiler bildet dabei neben einer guten Hautpflege die Applikation topischer Kortikoide. Durch die ständige Weiterentwicklung von Standardsubstanzen verfügen wir heute über wirksame und gleichzeitig gut verträgliche Therapeutika. So führt beispielsweise Mometasonfuroat (Ecural ${ }^{\circledR}$ ), ein topisches Kortikoid der Wirkstärkegruppe III, selbst unter einer einjährigen Dauerbehandlung zu keiner klinisch relevanten Hautatrophie.

In über zwei bis drei Wochen laufenden klinischen Studien mit diesem modernen Glukokortikoid wurde bei bis zu 9o\% der Patienten wieder ein normales Hautbild erzielt. Auch eine Kontaktallergie - wie man sie unter einigen Kortikoiden paradoxerweise zunehmend beobachten kann - wurde unter Mometason bisher nicht gesehen.

Speziell gegen den Juckreiz helfen orale Antihistaminika. Hier können auch die neueren nicht-sedierenden Substanzen verwendet werden, die teilweise auch eine offizielle Zulassung für diese Indikation haben. Bei stärkerer Symptomatik kommen zusätzlich UVB- oder UVA-Bestrahlungen sowie das Immunsuppressivum Ciclosporin zum Einsatz.

Ruzicka T

Pressesymposium der Essex Pharma, Petersberg bei Königswinter, August 2000. 